\title{
Comparison of the efficacy, safety, acceptability and fetomaternal outcomes of combination of mifepristone and foley's catheter with foley's catheter alone in induction of labour in term pregnancies with previous lower segment caesarean section
}

\author{
Nancy Thind, Pranav Sood, Rajeev Sood, Geetika Gupta Syal*
}

Department of Obstetrics and Gynecology, IGMC, Shimla, Himachal Pradesh, India

Received: 29 July 2020

Accepted: 05 September 2020

\author{
*Correspondence: \\ Dr. Geetika Gupta Syal, \\ E-mail: geetikaguptasyal@gmail.com
}

Copyright: $($ the author(s), publisher and licensee Medip Academy. This is an open-access article distributed under the terms of the Creative Commons Attribution Non-Commercial License, which permits unrestricted non-commercial use, distribution, and reproduction in any medium, provided the original work is properly cited.

\begin{abstract}
Background: Objective of the study was to compare the efficacy, safety, acceptability, fetomaternal outcomes of combination of mifepristone and Foley's catheter with Foley's catheter alone in induction of labor in term pregnancies with previous Lower segment caesarean section (LSCS).

Methods: This was a prospective study of 36 women induced with mifepristone and foley's catheter and 36 women induced with foley's catheter alone at 37 weeks to $41+6$ weeks with previous LSCS.

Results: Mean bishop score on admission in combined group (2.44) was comparable with that of foley's alone group (2.91, $\mathrm{p}=0.888$ ). Mean Bishop score (BS) after foley's expulsion in group A and group B was 7.46 and 6.33 respectively, which was statistically significant $(\mathrm{p}<0.001)$. In group A $69.5 \%$ of women delivered vaginally compared to $52.2 \%$ in group $B$ which was comparable $(\mathrm{p}=0.230)$. Mean induction to delivery interval was significantly short in combination group (15.5 \pm 1.3 hours versus $20.8 \pm 1.07$ hours, $\mathrm{p}=0.003)$. $50 \%$ women in group A required oxytocin for induction/ augmentation of labour as compared to $77.8 \%$ in group $B(p=0.02)$. Failed induction was statistically higher in group B $(\mathrm{p}<0.05)$. No difference was found with regards scar dehiscence, scar rupture, Postpartum hemorrhage $(\mathrm{PPH})$, wound infection, puerperal pyrexia, Meconium stained liquor (MSL), fetal distress, mean birth weight, 1 and 5 minutes Appearance, pulse, grimace, activity, and respiration (APGAR) score, neonatal outcome, hospital stay.

Conclusions: Priming with mifepristone before insertion of foley's catheter results in significant change in BS signifying that combination promotes early cervical ripening as compared to foley's catheter alone. Mifepristone plays significant role in cervical ripening, reduces induction to delivery interval, oxytocin requirement and failed induction.
\end{abstract}

Keywords: Bishop score, Foley's catheter, Induction of labor, Mifepristone, Previous LSCS

\section{INTRODUCTION}

Dr Edwin Cragin's, 1916 dictum of “Once a cesarean, always a cesarean" was used to be practiced by many obstetricians. For most of the 20th century caesarean section (CS) was a rarely used procedure. The caesarean rate hovered between $1-5 \%$ until about $1970 .^{1}$
Changes in technique, technology, good antibiotics, blood transfusions, Lower segment caesarean section (LSCS) improved the outcomes. ${ }^{2}$

The rate has risen in dramatic fashion from $<5 \%$ in the 1960 s to nearly $30 \%$ by $2004 .^{3}$ As the caesarean rate went up, that dictum, once a caesarean always a caesarean, 
became more of an issue. Therefore, any efforts to reduce the number of CS need to be reviewed. ${ }^{4}$

\section{METHODS}

The study was conducted in the Department of Obstetrics and Gynaecology at Kamla Nehru Hospital, Shimla from August 2017 to July 2018 which included women with singleton pregnancy (37-41+6 weeks), cephalic presentation, previous one LSCS, Bishop's score (BS) $<6$, consent for Trial of labor after caesarean (TOLAC).

\section{Exclusion criteria}

Patients with $\geq 2$ previous LSCS/classical CS/inverted T shaped CS/myomectomy scar, non-vertex presentation, lower segment width thickness (LSWT) $\leq 3 \mathrm{~mm}$, ruptured membranes/evidence of chorioamnionitis, obstetrical contraindication for vaginal birth (placenta previa, abruptio, cervical fibroid, Cephalopelvic disproportion (CPD), inter-delivery interval $\leq 18$ months).

After satisfying the inclusion and exclusion criteria women were counselled for Induction of labor (IOL), TOLAC and its risks. Written consent was obtained. Preinduction BS was recorded. On admission, patients were divided in two groups alternatively:

\section{Group A}

After receiving a single oral dose $400 \mathrm{mg}$ mifepristone, patients were reassessed after 48 hours, $16 \mathrm{~F}$ foley's catheter was inserted through internal ostium (under aseptic precautions) and filled with $40 \mathrm{ml}$ of NS. Catheter was then pulled against ostium and tapped to inner side of thigh. Patients were observed for 12 hours and then BS was reassessed. It was reassessed earlier if foley's catheter was expelled $<12$ hours.

\section{Group B}

After taking consent and BS assessment foley's catheter was introduced in same manner. Patients were observed for 12 hours and then BS was reassessed. It was reassessed earlier if catheter was expelled $<12$ hours.

If at any time $\mathrm{BS}>8$, IOL with amniotomy \pm oxytocin. All women were managed as per labour room protocols. Failure of induction was defined as failure to initiate labor after 12 hour of oxytocin infusion.

Both groups were compared with respect to change in BS, oxytocin requirement, mode of delivery, inductiondelivery interval (IDI) and feto-maternal outcome.

The intra operative findings (indication of CS, scar dehiscence, blood loss, blood transfusion, peripartum hysterectomy), and subsequent hospital stay was recorded in all the women who underwent CS for whatsoever indication. Neonatal outcome was observed (baby weight,
Apgar score at 1 and 5 minutes, neonatal intensive care unit (NICU) admission).

\section{Ethical clearance}

Ethical committee approval was obtained from the institute's ethical committee.

\section{Statistical analysis}

Appropriate statistical software and tools were used for analyzing. $p<0.05$ was considered as statistically significant.

\section{RESULTS}

Total number of deliveries during the study period was 6533 out of which 585 had previous caesarean. Amongst these 585 patients 354 had Elective repeat CS (ERCS) (99 women had previous two or more CS; 79 women had extension of previous uterine incision; and 176 had repeat CS for obstetrical indication) and of the remaining 231 eligible women, 58 refused TOLAC and 101 had spontaneous onset of labour pains. Hence, 72 women enrolled in the study were randomized in two groups (combined mifepristone and foley's catheter group (A), and foley's catheter alone group, (B)) with 36 women in each group.

Mean age, Body mass index (BMI) and gravidity were comparable in both groups.

Out of the total subjects, $9(25 \%)$ had previous history of Vaginal delivery (VD) in group A whereas $10(27.7 \%)$ had history of VD in group $B(p=0.561)$.

Majority of subjects were at gestational age of 39-41 weeks in both the groups i.e.72.2\% and $52.8 \%$ which was nonsignificant.

Inter-delivery interval was comparable $(<18 \mathrm{~m}$ was excluded).

Indications of $\mathrm{CS}$ in previous pregnancies in both the groups was tabulated. Failed induction $(16.7 \%$ versus $11.1 \%$, $\mathrm{p}=0.73)$, fetal distress $(41.7 \%$ versus $38.9 \%$, $\mathrm{p}=0.81)$, malpresentation $(30.6 \%$ versus $33.3 \%$, NS $)$, NPOL (11.1\% versus $16.7 \%$, NS)

Mean BS on admission in group A and group B was comparable (2.44 versus $2.91, \mathrm{p}=0.88)$. BS statistically improved after mifepristone $(5.8, \mathrm{p}<0.001)$.

Mean BS after foley's expulsion/removal in group A and group B was 7.46 and 6.33 respectively, which was highly significant $\quad(\mathrm{p}<0.001)$. Thus, prior priming with mifepristone before Foley's insertion resulted in significant change in BS signifying that combination promoted early cervical ripening (Table 1). 
Table 1: Bishop score comparison between the groups.

\begin{tabular}{|llllll|}
\hline Group & $\begin{array}{l}\text { BS on } \\
\text { admission } \\
\text { (mean } \pm \text { SD) }\end{array}$ & $\begin{array}{l}\text { BS after 48 hours } \\
\text { of mifepristone } \\
\text { (mean } \pm \text { SD) }\end{array}$ & $\begin{array}{l}\text { p } \\
\text { value }\end{array}$ & $\begin{array}{l}\text { BS after expulsion/ } \\
\text { removal (mean } \pm \text { SD) }\end{array}$ & \begin{tabular}{c} 
p value \\
\hline $\begin{array}{l}\text { Group A (Mifepristone } \\
\text { plus Foley's) }\end{array}$
\end{tabular} \\
\hline $\begin{array}{l}\text { Group B } \\
\text { (Foley's) }\end{array}$ & $2.44 \pm 0.92$ & $5.80 \pm 0.78$ & $<0.001$ & $7.46 \pm 1.23$ & $<0.001$ \\
\hline p value & $2.91 \pm 0.92$ & - & - & $6.33 \pm 0.82$ & $<0.001$ \\
\hline
\end{tabular}

Table 2: Distribution of cases according to mode of delivery.

\begin{tabular}{|c|c|c|c|}
\hline Mode of delivery & $\begin{array}{l}\text { Group A } \\
\mathbf{n}=36(\%)\end{array}$ & $\begin{array}{l}\text { Group B } \\
n=36(\%)\end{array}$ & p value \\
\hline Vaginal delivery & $25(69.5 \%)$ & $19(52.2 \%)$ & \multirow{2}{*}{0.230} \\
\hline CS & $11(30.5 \%)$ & $17(47.8 \%)$ & \\
\hline
\end{tabular}

Table 3: Previous vaginal delivery and its relation to VBAC success among groups.

\begin{tabular}{|c|c|c|c|c|c|}
\hline \multirow[t]{2}{*}{ Previous VD } & \multicolumn{2}{|c|}{ Successful VBAC $(n=44)$} & \multicolumn{2}{|c|}{$\begin{array}{l}\text { Caesarean section } \\
(\mathrm{n}=28)\end{array}$} & \multirow[t]{2}{*}{ p value } \\
\hline & Group A (\%) & Group B (\%) & Group A (\%) & Group B (\%) & \\
\hline Yes $(n=19)$ & $7(77.8)$ & $8(80)$ & $2(22.2)$ & $2(20)$ & 0.905 \\
\hline No $(n=53)$ & $18(48.6)$ & $11(42.3)$ & $19(51.4)$ & $15(57.7)$ & 0.875 \\
\hline
\end{tabular}

Table 4: Mode of onset of labour.

\begin{tabular}{|lll|}
\hline Study & BS at admission & BS after Foley's expulsion/removal \\
\hline${\text { Meetei et } \mathbf{~ a l}^{\mathbf{6}}}^{\mathbf{1 4}}$ & $2.51 \pm 1.1$ & $5.54 \pm 1.23$ \\
\hline${\text { Rezk et } \mathbf{l}^{\mathbf{1 4}}}^{\mathbf{7}}$ & $3.90 \pm 0.84$ & $5.95 \pm 0.87$ \\
\hline Cheuk et al $^{\mathbf{7}}$ & 3.0 & 7.0 \\
\hline Sharma et al & $\mathbf{1 2}$ & $5.7 \pm 1.6$ \\
\hline Present study & $2.0 \pm 1.4$ & \\
\hline Group A & & $7.46 \pm 1.23$ \\
\hline Group B & $2.44 \pm 0.92$ & $6.33 \pm 0.82$ \\
\hline
\end{tabular}

The CS rate in group A was less compared to group B, $30.5 \%$ versus $47.8 \%$, but was NS $(\mathrm{p}=0.230)$.

In this study, out of 72 women, 19 women had previous history of VD. Among these 19 patients, 15 (78.95\%) delivered vaginally and $4(21.05 \%)$ required repeat CS due to various indications which was statistically significant $(\mathrm{p}=0.04)$. Thus, previous VD was a good predictor of successful Vaginal birth after caesarean (VBAC) (Table 2).

In present study, 9 women had history of vaginal delivery in group A out of which 7 (77.8\%) delivered vaginally whereas in group B, 10 women had history of vaginal delivery out of which $8(80 \%)$ delivered vaginally this was comparable $(\mathrm{p}=0.905)$ showing that mifepristone addition does not influence the mode of delivery.
In group A, 13 women $(30.5 \%)$ had labour onset during ripening phase ( 5 with mifepristone only and 8 following foley's insertion) whereas only $5(13.8 \%)$ women had labour onset in group B (following foley's insertion) which was significant $(\mathrm{p}<0.05)$ (table 3$) .70 \%$ women in group B required oxytocin for IOL as compared to $39 \%$ in A which was significant $(\mathrm{p}=0.01)$ (Table 4$)$.

Induction delivery interval (IDI) was calculated from the insertion of foleys catheter to the vaginal delivery. In group A, 25 cases delivered vaginally. However only 20 required induction with foley's catheter following pretreatment with mifepristone in this group, as 5 cases had spontaneous onset of labour within 48 hours of mifepristone. The remaining 14 had CS due to various reasons. In group B, 19 delivered vaginally. Mean IDI in group A was significantly short $(15.5 \pm 1.31$ hours versus 20.8 \pm 1.07 hours, $\mathrm{p}=0.003$ ). 
A total $18(50 \%)$ women in group A required oxytocin for induction/augmentation compared to $28(77.8 \%)$ in group B which was significant $(\mathrm{p}=0.01)$.
Caesareans due to failed induction were significantly lower in group A ( 2 versus 9, p=0.049). Whereas all other indications (fetal distress, 5 versus 4; NPOL, 3 versus 2; scar dehiscence, 1 versus 2 ) were comparable.

Table 5: Changes in Bishop score.

\begin{tabular}{|llll|}
\hline Method & Group A $\mathbf{n = 3 6}(\%)$ & Group B $\mathbf{n = 3 6}(\%)$ & p value \\
\hline Mifepristone & $5(14 \%)$ & NA & \\
\hline Foleys catheter & $8(22 \%)$ & $5(14 \%)$ & 0.288 \\
\hline AROM & $9(25 \%)$ & $6(16 \%)$ & 0.293 \\
\hline Oxytocin & $14(39 \%)$ & $25(70 \%)$ & 0.01 \\
\hline
\end{tabular}

Table 6: Change in Bishop score after mifepristone.

\begin{tabular}{|lll|}
\hline Study & BS at admission & BS after mifepristone \\
\hline Athawale et al $^{\mathbf{1 1}}$ & $<3(84 \%)$ & $4-8(28 \%)$ \\
& $>3(16 \%)$ & $>8(72 \%)$ \\
\hline Acharya et al ${ }^{\mathbf{1 5}}$ & $2.34 \pm 0.84$ & $8.24 \pm 1.65$ \\
\hline Sharma et al $^{\mathbf{1 2}}$ & $2.0 \pm 0.84$ & $3.5 \pm 1.7$ \\
\hline Present study & $2.44 \pm 0.92$ & $5.80 \pm 0.78$ \\
\hline
\end{tabular}

Both the groups were comparable in terms of approximate blood loss, PPH, wound infection, 1 and 5 minutes. Appearance, pulse, grimace, activity, and respiration (APGAR) score, birth weight, perinatal morbidity, mortality and hospital stay.

\section{DISCUSSION}

This study was conducted to compare the efficacy and safety of combination of mifepristone and intra-cervical foley's catheter with conventional use of foley's catheter alone on cervical ripening and labour induction in women with previous one LSCS with a goal to reduce the rates of ERCS and ultimately overall caesarean rates.

In the present study previous VD rates were $25 \%$ and $27.7 \%$ in group A and group B respectively which was comparable ( $\mathrm{p}=0.561)$ just like $22.72 \%$ in study by Sharma et al5, $13.3 \%$ in Meetei et al6 and $16.6 \%$ in Cheuk et al. ${ }^{7}$ In study by Madaan et a, $\mathrm{p}=0.007$, Landon et al, $\mathrm{p}<0.001$ it was shown that previous VD was a good positive predictor for VBAC. ${ }^{8,9}$ Since the VD rates were comparable in both the groups in our study we could compare further results without influence of this history.

In all the studies majority of the patients underwent intervention after 39 weeks which was in accordance with FOGSI-ICOG 2018 guidelines. ${ }^{10}$ Athawale et al 39.29 \pm 0.9 weeks, Meetei et al 38 weeks, Cheuk et al 39.1 weeks, present study group A- 39.4 weeks and group B- 39 weeks. ${ }^{6,7,11}$

Most common indication of CS in previous pregnancy in most of the studies including our study was fetal distress and malpresentation which was comparable in both groups $\mathrm{p}=0.8$. Previous CS done in our study for fetal distress in group $\mathrm{A}$ and $\mathrm{B}$ was $41.7 \%$ and $38.9 \%$ respectively comparable to Sharma et al $36 \%$, Hemalatha et al $36.6 \% .^{12,13}$ Previous CS done in our study for malpresentation was $30.6 \%$ and $33.3 \%$ in the two groups comparable to Sharma et al $30 \%$, Hemalatha et al $13.3 \% .^{12,13}$

In all these studies including the present study change in BS was found to be significant (Table 5).

In all these studies change in BS was found to be significant after mifepristone except the study by Sharma et al probably because in this study assessment was done after 24 hours unlike other studies (48 hours) (Table 6). ${ }^{12}$

In the present study $36 \%$ subjects in group A and $14 \%$ subjects in group B went into labour during the ripening phase. Group B was comparable to the study by Meetei et al (who had kept Foley's for 12 hours). ${ }^{6}$ St Onge et al and Sharma et al showed better results because foley's catheter was kept for longer duration i.e. 24 hours. ${ }^{12,17}$ In the present study in group A where mifepristone was also used along with foleys catheter, $36 \%$ subjects went in labour as compared to $14 \%$ subjects in group B, this was statistically significant $(\mathrm{p}=0.049)$. This was also observed in study by Acharya et al, where $14 \%$ subjects entered in active phase of labour with mifepristone only and Sharma et al where $56 \%$ women had labour onset within 48 hours of mifepristone administration. ${ }^{15,12}$ Thus, it is concluded that in addition to Foleys catheter prior ripening with mifepristone had better success rate in IOL.

In the present study, in group A there was a statistically significant shortening of IDI as compared to group B 
( $\mathrm{p}=0.049)$. Group B showed IDI 20.8 \pm 1.07 hours which was comparable to Cheuk et al (19 hours), Hemlatha et al (23 hours), Ziyauddin et al (18.5 hours) and Rezk et al (22.2 hours). In all these studies foley's catheters was used for IOL. ${ }^{7,14,13,16}$ Sharma et al showed IDI of 50.9 hours in patients induced with foley's catheter which may be due to the fact that foley's catheter was kept for longer duration i.e. 24 hours. ${ }^{12}$ In group A combination of mifepristone with foley's catheter resulted in a shorter IDI. This could not be compared as there is no such study found in literature. Thus, it was observed that combination of mifepristone and foleys catheter has better results with regards to decrease in IDI.

In present study, 50\% subjects required oxytocin for induction/augmentation of labour in group A versus $78 \%$ in group $B(p=0.02)$. The results of group $B$ were similar to the studies by Cheuk et al where oxytocin augmentation was required in $75 \%$ of cases and Sharma et al where it was required in $78 \%$ of cases. ${ }^{7,12}$ In the present study it was observed that when mifepristone was combined with foley's catheter, oxytocin was required only in 50\% subjects. Hence, combination of mifepristone with foleys catheter significantly reduces oxytocin requirement $(\mathrm{p}=0.02)$.

The success rate of VBAC in various studies using foley catheter ranged from $40 \%$ (NS) in study by Sharma et al to a maximum of $66.7 \%$ (significant) in study by Meetei et al. ${ }^{6,12}$ In our study the success rate of VBAC was $69.5 \%$ in group $\mathrm{A}$ and $52.2 \%$ in group $\mathrm{B}$. The difference in the results could be due to different institutes having different threshold for CS in a previously scarred uterus. Though the success rate of VBAC was more in group A as compared to group B but it was NS ( $\mathrm{p}=0.230)$.

In the present study 5\% had CS for failed induction in group A and 25\% in group B. Hemaltha et al and Sharma et al each showed $16 \%$ rate. ${ }^{12,13}$ There was a significant reduction of $\mathrm{CS}$ done for failed induction in group $\mathrm{A}$ as compared to group B ( $\mathrm{p}=0.049)$. These observations are in accordance with Cochrane systematic review on mifepristone for IOL which stated that it was better than placebo in reducing the rates of CS performed for failed induction. ${ }^{12}$

NPOL was seen in $8 \%$ and $5 \%$ cases in group A and B respectively. This was comparable within groups and to the studies by Hemlatha et al $(6 \%)$ and Cheuk $(8 \%)^{7,13}$ Fetal distress was seen in $14 \%$ in group A and $11 \%$ in group B. This was similar to results by Cheuk et al (13\%) and Sharma et al (18\%). ${ }^{7,12}$ Scar dehiscence was an indication for CS in group A in 2\% and in group B in 5\% patients. This was similar to the studies by Cheuk et al (4\%) and Sharma et al (8\%).,12 Hence addition of mifepristone has not influenced the rates of CS for the indications of NPOL, fetal distress and scar dehiscence.

\section{CONCLUSION}

We concluded from our study that addition of mifepristone prior to intracervical foley's catheter insertion improves the outcome with regards to induction by producing more favourable change in BS when compared to foley's catheter alone. Further the IDI and the oxytocin requirement decreases when mifepristone is used for cervical ripening before Foley's catheter insertion. However, there is no significant change in mode of delivery and fetomaternal outcomes. Hence mifepristone appears to be safe, efficient and suitable agent for cervical ripening and for initiation of labor when given 48 hours prior to labor induction with foley's catheter.

\section{Funding: No funding sources}

Conflict of interest: None declared

Ethical approval: The study was approved by the Institutional Ethics Committee

\section{REFERENCES}

1. Flamm BL. Once a cesarean, always a controversy. Obstet Gynecol. 1997;90(2):312-5.

2. Cypher RL. Cesarean Birth: A Journey in Historical Trends. J Perinat Neonatal Nurs. 2016;30(3):259-64.

3. Niino $Y$. The increasing cesarean rate globally and what we can do about it. Biosci Trends. 2011;5(4):139-50.

4. Arulkumaran S. Caesarean section rates are increasing worldwide. Preface. Best Pract Res Clin Obstet Gynaecol. 2013;27(2):151-2.

5. Sharma C, Soni A, Soni PK, Verma S, Verma A, Gupta A. A Retrospective Case-Control Study Evaluating the Role of Mifepristone for Induction of Labor in Women with Previous Cesarean Section. J Obstet Gynaecol. 2016;66(1):30-7.

6. Meetei LT, Suri V, Aggarwal N. Induction of labor in patients with previous cesarean section with unfavorable cervix. J Med Soc. 2014;28(1):29-33.

7. Cheuk QKY, Lo TK, Lee CP, Yeung APC. Double balloon catheter for induction of labour in Chinese women with previous caesarean section: one-year experience and literature review. Hong Kong Med J Xianggang Yi Xue Za Zhi. 2015;21(3):243-50.

8. Madaan M, Agrawal S, Nigam A, Aggarwal R, Trivedi SS. Trial of labor after previous caesarean section: the predictive factors affecting outcome. J Obstet Gynecol. 2011;31:224-8.

9. Landon MB, Leindecker S, Spong CY, Hauth JC, Bloom S. The MFMU Cesarean Registry: Factors affecting the success of trial of labor after previous cesarean delivery. Am J Obstet Gynecol. 2005;193:1016-23.

10. FOGSI-ICOG 2018. Induction of Labor: Good Clinical Practice Recommendations. Last accessed on 1 st December 2018.

11. Athawale R, Acharya N, Samal S, Hariharan C. Effect of mifepristone in cervical ripening for induction of 
labour. Int J Reprod Contracept Obstet Gynecol. 2016;2(1):35-8.

12. Sharma C, Soni A, Gupta A, Verma A, Verma S. Mifepristone vs balloon catheter for labor induction in previous cesarean: a randomized controlled trial. Arch Gynecol Obstet. 2017;297(1):77-83.

13. HK R, DS. Case series of Foley's induction in patients with previous caesarean. Int $\mathbf{J}$ Reprod Contracept Obstet Gynecol. 2017;6(4):1635.

14. Rezk M, Sanad Z, Dawood R, Masood A, Emarh M, Halaby AA. Intracervical Foley Catheter Versus Vaginal Isosorbid Mononitrate for Induction of Labor in Women With Previous One Cesarean Section. J Clin Gynecol Obstet. 2014;3(2):55-61.
15. Acharya R, Chaudhari P, Choudhary A, Sharma A, Jain S. Mifepristone as cervical ripening agent for Labour induction in women with Previous one Caesarean Section. Int J Med Res Rev. 2016;4(4):6249.

Cite this article as: Thind N, Sood P, Sood R, Syal GG. Comparison of the efficacy, safety, acceptability and fetomaternal outcomes of combination of mifepristone and foley's catheter with foley's catheter alone in induction of labour in term pregnancies with previous lower segment caesarean section. Int J Reprod Contracept Obstet Gynecol 2020;9:4181-6. 